\title{
Commentary: Can you use a hammer to pound a nail? Are there fish in the sea?
}

\author{
Danielle O'Hara, BSc, ${ }^{a}$ and Joanna Chikwe, $\mathrm{MD}^{\mathrm{a}, \mathrm{b}}$
}

\footnotetext{
From the ${ }^{\mathrm{a} D e p a r t m e n t}$ of Surgery, The State University of New York, Stony Brook, NY; and ${ }^{\mathrm{b}}$ Department of Cardiovascular Surgery, Mount Sinai Medical Center, New York, NY.

Disclosures: Authors have nothing to disclose with regard to commercial support.

Received for publication May 16, 2019; accepted for publication May 16, 2019; available ahead of print Aug 16, 2019 .

Address for reprints: Joanna Chikwe, MD, Department of Cardiovascular Surgery, Mount Sinai Medical Center, 1190 Fifth Ave, New York, NY 10029 (E-mail: Joanna.Chikwe@mountsinai.org).

J Thorac Cardiovasc Surg 2020;159:192-3

$0022-5223 / \$ 36.00$

Copyright (c) 2019 Published by Elsevier Inc. on behalf of The American Association for Thoracic Surgery

https://doi.org/10.1016/j.jtcvs.2019.05.029
}

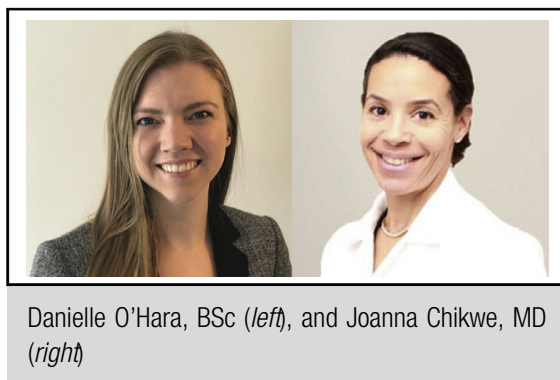

\section{Central Message}

Prolonged intubation is usually a marker rather than a cause of sicker patients and of adverse events, including delirium.

See Article page 182 their analysis of data from 2561 patients at a single center between 2010 and 2015, Muller Moran and colleagues ${ }^{1}$ identified delirium twice as frequently (28\%) in the $13 \%$ of patients extubated 12 to 24 hours postoperatively, compared to in $12 \%(P<.001)$ of patients extubated within 12 hours, who also tended to be younger with less comorbidity. The adjusted odds of delirium increased approximately $10 \%$ per hour in patients intubated longer than 12 hours. Muller Moran and colleagues ${ }^{1}$ conclude $^{2}$ that "delirium rates increase with lengthier postoperative ventilation times" and that this is a basis for considering "earlier extubation to minimize delirium."

Is this conclusion reliable? Delirium was identified prospectively, as assessed by a validated, structured questionnaire that was performed 4 hourly to identify confusion. This prospective approach is one of the strengths of this study. It also likely explains the relatively high delirium rate in this otherwise low-risk cohort. This is firstly because the responses of many surgeons, let alone postoperative patients, if woken repeatedly overnight to answer this questionnaire would probably qualify as delirium by these criteria. Second, the questionnaire requires excellent English language skills to administer and answer correctly. Standard questions include, "Are there fish in the sea?" and "Can you use a hammer to pound a nail?" Failure to reply yesincluding completely logical answers such as "No, I've never been able to hit a nail straight," "No, not right now," "No, I don't understand," or even "No, go away, I'm trying to sleep" - count toward a delirium score.

An additional limitation is the scantiness of the data on perioperative causes of both prolonged intubation and delirium. For example, anesthetic technique and sedation, analgesia, and weaning protocols are the primary drivers of both extubation times and cognitive function in most patients undergoing uncomplicated coronary artery bypass grafting. Failing to analyze these factors is problematic, first, because it prevents us from understanding whether the association between prolonged intubation and delirium could be explained by excessive long-acting narcotics or other agents, and second, because it prevents us from identifying potential targets for practice improvement. Similarly, intraoperative and early postoperative problems, such as stroke, shock, and metabolic compromise, may result in both prolonged intubation and delirium, so it is unfortunate that these factors were not included in the multivariate analysis.

Consequently, the value of this study is limited to documenting the incidence of delirium after coronary artery bypass grafting. These data will not change our practice of extubation within 6 hours for the vast majority of our patients undergoing coronary artery bypass grafting, supported by anesthesia and nursing protocols designed to optimize analgesia, early mobilization, and undisturbed sleep. The few patients needing more than 12 hours for extubation after coronary artery bypass grafting almost invariably have more significant medical problems dictating the speed of safe weaning 
from the ventilator. Prospective studies, such as the trial of haloperidol versus placebo recently reported in The New England Journal of Medicine, may eventually help us to reduce the risk of delirium in these patients, for whom earlier extubation is rarely the safest option. ${ }^{2}$

\section{References}

1. Muller Moran HR, Maguire D, Maguire D, Kowalski S, Jacobsohn E, Mackenzie S, et al. Association of earlier extubation and postoperative delirium after coronary artery bypass grafting. J Thorac Cardiovasc Surg. 2020;159:182-90.e7.

2. Girard TD, Exline MC, Carson SS, Hough CL, Rock P, Gong MN, et al. Haloperidol and ziprasidone for treatment of delirium in critical illness. $N$ Engl J Med. 2018;379:2506-16 